\title{
ВЛИЯНИЕ ВЫПУСКА КОНЕЧНОГО ПРОДУКТА НА ЦЕНУ АКЦИИ КОМПАНИИ ПРОИЗВОДИТЕЛЯ
}

А.В. Соломатин ${ }^{1}$, Я.В. Соломатин ${ }^{2}$

В данной работе изучается, как выпуск конечного продукта на рынок (релиз компьютерной игры) может повлиять на цену акции ее разработчика/издателя. Были получены данные по 69 компьютер-ным играм, выпущенные в период 2004-2013 гг., а также финансовая информация о 9 публичных компаниях, которые разработали/издали данные игры. После чего данные были обработаны с помощью двух различных методологий. В обоих случаях было выяснено, что релиз компьютерной игры имеет положительный, но кратковременный (до 48 часов) эффект на цену акции компаниишпроизводителя. Так же было выяснено, что больший эффект достигается если качество выпущенной игры превзошло ожидания игроков.

Ключевые слова: Цена, акция, доходность, рынок ценных бумаг, продукт, капитализация.

JEL: G11

\section{Введение}

Выпуск конечного продукта на рынок (в нашем случае релиз компьютерной игры) является важным событием как для ее разработчика, так и для издателя. Некоторые игры разрабатываются в течение -многих лет (например, Half-Life 2, Duke Nukem Forever и т.д.), иногда определяя дальнейший успех mфирмы. Согласно Baker (2011), инвесторы принимают во внимание информацию о выпуске игры ғпри торговле акциями фирм-разработчиков видеоигр. Однако, несмотря на это, не было найдено ни 之одного академического исследования по этой теме. Это серьезный пробел, который будет отчасти чустранен в данной работе.

ㄱзльтаты данного исследования представляют интерес для трех типов агентов: (1) инвесторов, которые смогут улучшить прогноз цен акций и получить дополнительную прибыль; (2) владельцев/ топ-менеджеров фирм по разработке/изданию компьютерных игр - они выяснят, как ожидания потребителей могут повлиять на капитализацию их компании; (3) потребителей/игроков - если они узнают, что их мнение оказывает значимое влияние на фирму, то они будут мотивированы чаще давать обратную связь разработчикам.

В первую очередь будет подробно рассмотрен тип исследования. После этого описаны полученные данные и методология составления финальной выборки. Затем будут рассмотрены и применены два подхода к оценке влияния рассматриваемого события на цену акции, а также полученные с помощью данных подходов результаты и рекомендации для инвесторов. В завершение рассмотрены недостатки исследования и сделаны выводы.

\section{Общий тип исследования}

В данной работе применяется количественный (корреляционный) тип исследования. Он подходит наилучшим образом для данной темы по нескольким причинам.

Во-первых, основной целью данной работы является тестирование причинно-следственных связей, и ни один другой тип исследования не может дать нам столь точные инструменты для выполнения этой задачи. Во-вторых, используя данный тип исследования, будет легче найти подходящую методологию для тестирования влияния релиза игры, так как большинство статей по событийному анализу ${ }^{3}$ применяют именно корреляционный тип исследования. В-третьих, исследованию необходимо обладать высокой внешней валидностью (так как предполагается, что работа может быть использования инвесторами для прогнозирования цен акций разработчиков/издателей компьютерных

1. Негосударственное образовательное учреждение «Московская международная высшая школа бизнеса «МИРБИС» канд. экон. наук, проректор по научной работе.

2. Закрытое акционерное общество Коммерческий банк «Ситибанк», Аналитик отдела развития продукта депозитарного управления, DCC Analyst.

3. Общее название исследований, которые изучают влияние какого-либо события на определенный параметр, в основном на цену акции. 
игры) - корреляционный тип исследования обладает подобным преимуществом благодаря большому количеству исследуемых наблюдений.

\section{Данные и выборка}

Данное исследование полностью основано на вторичных данных. Вся финансовая информация (цена акций, биржевые индексы, бухгалтерские коэффициенты и т.д.) была получена из Bloomberg; информация о дате релиза игр была взята с официальных сайтов разработчиков/издателей. Данные о качестве игр были взяты с сайта Metacritic (качество оценивается по 100-балльной системе).

В первую очередь была использована база данных информационного агенства Bloomberg для того, шчтобы найти все публичные компании, разрабатывающие/выпускающие компьютерные игры. Затем на этот список фирм были наложены три ограничения:

- Компания должна разработать/выпустить, по крайней мере, одну компьютерную игру в промежутке с 2004 по 2013 г.

- Акции компании должны торговаться по крайней мере в течение 500 дней во время описанного выше временного промежутка.

- Основной доход компании должен быть получен благодаря разработке/выпуску видеоигр.

Первое ограничение было сделано для того, чтобы избежать включения в выборку очень старых игр, которые были выпущены, когда рынок значительно отличался от сегодняшнего. Второе - для мтого, чтобы увеличить статистическую мощность тестов - если включить в выборку фирмы с чрезғвычайно неликвидными акциями, то очень вероятно, что взаимосвязь между ценой акции и рели일 игры выявлена не будет, даже если она существует. Последнее ограничение было наложено по той же причине: логично предположить, что для таких гигантов, как Microsoft или Sony (они так же Eразрабатывают и выпускают компьютерные игры), релиз игры не окажет значительного влияния на ळцену их акций, так как для данных фирм это событие не является значимым, в то время как, например, для Activision Blizzard релиз каждой игры чрезвычайно важен. Последнее ограничение просто разделяет эти два типа фирм.

После наложения ограничений в выборку попали только 18 фирм. Однако так как процесс обработки финансовых данных чрезвычайно трудоемкий, было принято решение случайным образом выбрать 9 фирм. Список фирм, акции которых были рассмотрены в данном исследовании, представлен в таблице 1.

Следующим шагом является выбор игр, которые были разработаны/изданы вышеописанными компаниями. Здесь так же накладываются три ограничения:

- Информация о дате релиза игры и ее качестве должна быть доступна

- Игра должна быть как минимум второй для рассматриваемой фирмы

- $\quad$ За 60 дней до и 10 дней после релиза игры рассматриваемая фирма не должна производить релиз какой-либо другой игры.

Первое ограничение накладывается для того, чтобы гарантировать наличие всей необходимой информации. Второе ограничение - для того, чтобы оценка ожидаемого качества игры была возможной${ }^{1}$. Третье ограничение было введено исключительно из-за особенностей используемой методологии - для качественной оценки влияния события на цену акции необходимо гарантировать, чтобы в этот промежуток времени не происходили иные подобные события [MacKinlay, 1997].

После всех ограничений в конечной выборке осталось 69 компьютерных игр (релизов). Полный список рассматриваемых игр представлен в Таблице 2. Можно удостовериться, что полученная выборка репрезентативна - в ней представлены игры разных жанров, из разных стран, с разным качеством и датой выхода. Это обеспечит высокую внешнюю валидность результатов работы.

1. По методу адаптивных ожиданий, который будет применен в данном исследовании,, ожидаемое качество игры зависит от реального и ожидаемого качества предыдущих игр фирмы. Если рассматриваемая игра - первая для фирмы, то оценка ожиданий не представляется возможной. 


\section{Подход 1}

\section{Методология}

В данном подходе будет использована методология, напоминающая обычный регрессионный анаблиз, и которая была широко рассмотрена в статье Биндер [Binder, 1998]. Для каждой игры поведение цены акции изучается в день релиза и в 21-й день после него (что примерно равно одному ка-

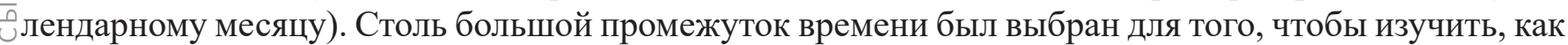
чдолго в среднем после релиза игры наблюдается чрезмерная доходность вследствие неэффектив工 шЗависимой переменной в регрессии для каждого дня является кумулятивный доход акции в этот гдень ${ }^{1}$. Она может быть представлена формулой:

$C R_{i}=\sum_{t=0}^{i} \frac{P_{t}-P_{t-1}}{P_{t-1}}$

где $C R_{\mathrm{i}}$ - кумулятивная доходность в і-й день после релиза игры $c$;

$P_{t}$ - цена закрытия в t-й день после релиза игры.

Кумулятивная доходность для 22 дней (включая день релиза) может быть представлена в виде маงтрицы:

\begin{tabular}{c|cccccc} 
& игра_1 & игра_2 & $\ldots$ & игра_n & $\ldots$ & игра_69 \\
\hline день_0 & $C R_{0}^{1}$ & $C R_{0}^{2}$ & $\ldots$ & $C R_{0}^{n}$ & $\ldots$ & $C R_{0}^{69}$ \\
день_1 & $C R_{1}^{1}$ & $C R_{1}^{2}$ & $\ldots$ & $C R_{1}^{n}$ & $\ldots$ & $C R_{1}^{69}$ \\
$\ldots$ & $\ldots$ & $\ldots$ & $\ldots$ & $\ldots$ & $\ldots$ & $\ldots$ \\
день_i & $C R_{i}^{1}$ & $C R_{i}^{2}$ & $\ldots$ & $C R_{i}^{n}$ & $\ldots$ & $C R_{i}^{69}$ \\
$\ldots$ & $\ldots$ & $\ldots$ & $\ldots$ & $\ldots$ & $\ldots$ & $\ldots$ \\
день_21 & $C R_{21}^{1}$ & $C R_{21}^{2}$ & $\ldots$ & $C R_{21}^{n}$ & $\ldots$ & $C R_{21}^{69}$
\end{tabular}

Где игра_n-компьютерная игра под номером $n$,

день_ $i-i$-й день после релиза игры

Для того чтобы спрогнозировать кумулятивную доходность для каждого дня, требуется принять во внимание не только информацию о реальном/ожидаемом качестве игры, но и проконтролировать иные переменные, которые могли бы повлиять на доходность акции. Поэтому для каждого дня выводится своя собственная регрессия.

Модель кумулятивной доходности для каждого дня имеет следующий вид:

$C R_{i}=\alpha+\sum_{t=1}^{n}\left(\beta_{t} X_{t}\right)+\varepsilon_{i}$

где $X_{t}$ - объясняющая переменная t (обсуждается далее),

$\varepsilon_{i}$ - ошибка для $i$-й регрессии

Данный подход имеет определенные ограничения, которые будут подробно рассмотрены в разделе «Недостатки».

\section{Оиенка объясняюших переменных. Реальное и ожидаемое качество игры}

Для событийного анализа классификация событий является частой практикой. Например, Болл и Браун [Ball and Brown, 1968] разделили объявления о доходах фирм на «хорошие» и «плохие» в зависимости от доходов в прошлом периоде. Поэтому в данном исследовании также необходимо разработать метод дискриминации изучаемых событий (которые в данном случае являются релизами игр).

1. Цена акции сама по себе не может являться зависимой переменной вследствие автокорреляции, нарушающей условия Гаусса-Маркова. 
Для этого необходимо определить: (1) каково истинное качество выпущенной игры; (2) каковы были ожидания о качестве выпущенной игры. Сравнивая эти два параметра, можно определить, какую информацию (негативную/нейтральную/позитивную) несет в себе релиз игры. К сожалению, универсального способа оценки этих параметров не существует - он должен быть создан.

Самым очевидным способом оценки качества игры является использование оценок с обзоров компьютерных игр. Данный метод кажется весьма надежным - в соответствии с Бейкер [Baker, 2011] 气 обзоры игр в значительной степени определяют поведение потребителей и могут спрогнозировать коммерческий успех игры. Кроме того, в исследовании Делоит [Deloitte, 2007] указывается, что обзор того или иного продукта повлиял на решение о покупке $82 \%$ респондентов.

В данном исследовании будет использована средняя экспертная оценка с сайта Metacritic. Этот исбтчник был выбран по нескольким причинам. Во-первых, он широко используется как учеными [Plucker et al., 2009; Situmeang et al. 2014], так и инвесторами [Baker, 2011]. Во-вторых, в нем содержатся оценки о большинстве компьютерных игр, соответственно вероятность пропустить непопулярную игру будет в рамках статистической погрешности, что увеличивает внешнюю валидность исследования. В-третьих, сайт предоставляет относительно объективную оценку качества игры благодаря агрегированию оценок множества экспертов, что увеличивает внутреннюю валидность работы.

Так как не было обнаружено ни одного источника данных, который бы предоставлял информацию об ожиданиях качества компьютерных игр, в исследовании делается предположение, что они (ожимдания) следуют модели адаптивных ожиданий:

$E\left(Q_{i}^{n}\right)=E\left(Q_{i-1}^{n}\right)+\lambda \times\left(Q_{i-1}^{n}-E\left(Q_{i-1}^{n}\right)\right) \forall i \neq 1, \lambda \in[0 ; 1]$

где $Q_{i}^{n}$ - реальное качество і-й по счету игры в фирме $n$,

$E\left(Q_{i}^{n}\right)$ - ожидаемое качество і-й по счету игры в фирме $n$,

$\lambda$ - коэффициент дисконтирования, который определяет, насколько сильно влияет разница между реальным и ожидаемым качеством предыдущих игр на ожидаемое качество данной игры. Здесь предполагается, что $\lambda=0.5$

Использование данной модели гарантирует постепенную и сбалансированную адаптацию ожиданий - если качество предыдущей игры в фирме оказалось слишком высоким/низким относительно ожиданий, то это не будет иметь ощутимых последствий на ожидания о качестве рассматриваемой игры. Однако если реальное качество многих игр в прошлом оказалось выше/ниже ожиданий, ожидания о качестве новой игры будут значительно изменены.

Модель адаптивных ожиданий также достаточно интересна, так как она отображает некоторые психологические аспекты поведения потребителей. Например, в Эрдем [Erdem, 1998] и Ситуминг и соавторы [Situmeang et al., 2013] утверждается, что потребители связывают свойства (в том числе и качество) предыдущих товаров, выпускаемых определенной фирмой, со свойствами нынешних. Андерсон [Anderson, 1973] и Оливер [Oliver, 2009] также утверждают, что успех предыдущих товаров увеличивает ожидаемое качество будущих товаров этой же фирмы. Все эти эмпирические результаты принимаются моделью адаптивных ожиданий во внимание.

\section{Выбор контрольных переменных}

Существует внушительный объем академической литературы, предлагающей различные параметры, которые могут быть использованы для предсказания дневной доходности акции. Например, Шолтенс и Ванг [Scholtens and Wang, 2008] определили, что доходность акции положительно коррелирована с доходностью рынка, ценой на нефть, но негативно с отношением балансовой стоимости фирмы к ее рыночной стоимости. Лаконишок [Lakonishok et al., 1994] утверждают, что информация о доходах, дивидендах и балансовой стоимости фирмы в прошлых периодах может быть успешно использована для прогнозирования доходности акции. Однако некоторые ученые поддерживают гипотезу об эффективном рынке и утверждают, что цену акции невозможно предсказать [Kim and 
Shamsuddin, 2008; Fama and French, 1993]. Поэтому выбор контрольных переменных является весьма спорным, так как какой бы набор параметров нь был бы выбран, он все равно может быть подвергнут критике. Кроме того, некоторые важные параметры могут быть не включены в модель из-за незнания, что приведет к ошибке невключенной переменной. Тем не менее в данной работе были рассмотрены наиболее доступные и важные параметры (полный список представлен в табл. 3).

Стоит заметить, что для всех финансовых параметров (коэффициент «Цена/прибыль», капитализация фирмы и т.д.) применялось не абсолютное значение, а его ежегодное изменение в процентах. †то было сделано потому, что по Коллиер [Collier, 2012] бухгалтерские коэффициенты и иная финансовая информация должна всегда с чем-то сравниваться: с ожидаемым значением, со значением этого параметра у конкурирующей компании или со значением из прошлых периодов. Соответственно в работе был выбран последний вариант из-за его доступности.

\section{Анализ данных}

После определения переменных и выборки, было сформировано информационное множество для каждого дня. Множество имеет следующий вид:

День X (см. табл. 1 для расшифровки сокращений)

зависимая переменная

\begin{tabular}{|c|c|c|c|c|c|c|}
\hline$C R_{x}^{1}$ & $\operatorname{indret}_{\mathrm{x}}^{1}$ & Cntret $_{x}^{1}$ & $\ldots$ & Expinde $_{x}^{1}$ & $\operatorname{Good}_{x}^{1}$ & $\operatorname{Bad}_{x}^{1}$ \\
\hline$C R_{x}^{2}$ & indret $_{x}^{2}$ & Cntret $_{x}^{2}$ & $\ldots$ & Expinde $_{x}^{2}$ & $\operatorname{Good}_{x}^{2}$ & $\operatorname{Bad}_{x}^{2}$ \\
\hline$\cdots$ & ... & ... & ... & ... & $\ldots$ & $\ldots$ \\
\hline$C R_{x}^{n}$ & indret $_{\mathrm{x}}^{\mathrm{n}}$ & Cntret $_{x}^{n}$ & $\ldots$ & Expinde $_{\mathrm{x}}^{\mathrm{n}}$ & $\operatorname{Good}_{\mathrm{x}}^{\mathrm{n}}$ & $\operatorname{Bad}_{x}^{n}$ \\
\hline$\ldots$ & ... & $\ldots$ & ... & ... & $\ldots$ & ... \\
\hline$C R_{x}^{69}$ & indret $_{x}^{69}$ & Cntret $_{\mathrm{x}}^{69}$ & $\ldots$ & Expindex ${ }_{x}^{69}$ & $\operatorname{Good}_{x}^{69}$ & $\operatorname{Bad}_{x}^{69}$ \\
\hline
\end{tabular}

Прежде чем получить конечную модель необходимо решить проблему мультиколлинеарности. Для этого из модели поочередно удаляются параметры с VIF (фактор инфляции дисперсии) выше пяти. Конечная модель для каждого дня имеет следующий вид:

$\mathbb{R}_{i}=\alpha+\beta_{1}$ indret $_{i}+\beta_{2}$ ñntret $_{i}+\beta_{3}$ prev_ret $_{i}+\beta_{4}$ PEchange $_{i}+\beta_{5}$ Pbkchange $_{i}+\beta_{6}$ Capchange $_{i}+$ $\beta_{7}$ Equitychang $_{i}+\beta_{8}$ EPSchange $_{i}+\beta_{9}$ Quality $_{i}+\beta_{0}$ Expindex $_{i}+\varepsilon$

В данной модели представляют интерес не только знак и значимость коэффициентов перед параметрами Quality $_{i}$ и Expindex ${ }_{i}$, но так же в какой степени включение данных параметров (которые представляют информацию о выпускаемой игре) в модель увеличивает качество ее прогноза. Для этого были созданы два типа моделей - одни включают в себя информацию об игре (Quality ${ }_{i}$ и Expindex $_{i}$ ), другие нет, а затем сопоставлены их коэффициенты детерминации.

\section{Результатьл}

Результаты регрессионного анализа представлены в Таблице 4 (см. Приложение).

Несмотря на тот факт, что малое количество коэффициентов оказались значимы, объясняющие возможности моделей оказались достаточно высокими - средний скорректированный коэффициент детерминации равен $46,1 \%$ с диапазоном от $31,9 \%$ до $55,4 \%$.

Коэффициент перед параметром «качество игры» оказался статистически значимым только в день релиза, в то время как индекс «ожидаемого качества игры» - в 19-й день после релиза. Однако это не столь важно. Из гистограммы 1 можно видеть, что в день релиза и на следующий день включение информации об ожидаемом/реальном качестве игры значительно увеличивает качество предсказания кумулятивного дохода акции. Следовательно, можно сделать вывод, что принятие во внимание информации о релизе игры может быть полезно для прогнозирования цены акции ее разработчика/издателя в день самого релиза и на следующий день; для последующих дней польза от 
подобной информации весьма незначительна. Стоит заметить, что только реальное качество игры, но не ожидаемое качество, оказалось статистически значимым. Это может происходить по двум причинам. Во-первых, возможно, на финансовом рынке почти никто не принимает во внимание ожидания игроков и поэтому разница между ожидаемым и реальным качеством (именно это и по亡 $\rightarrow$ казывает индекс ожиданий) становится бесполезной. Во-вторых, вполне возможно, что на самом деле ожидания важны, но в данной работе они не были смоделированы должным образом, что при-

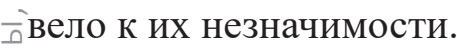

\section{Рекомендации для инвесторов}

Несмотря на тот факт, что в большинстве случаев параметры выпущенной игры оказались статиш стически незначимы, инвесторам рекомендуется принимать во внимание информацию о выпуске ๓ игр при спекуляции акциями разработчиков/издателей видеоигр в течение 48 часов после релиза. Если качество выпущенной игры оказалось высоким, то при прочих равных цена акции будет расти. Если после релиза игры прошло более двух торговых дней, то информация о ней, скорее всего, будет бесполезна.

\section{Подход 2}

В данном разделе будет представлен альтернативный подход к оценке влияния выпуска компьютерных игр на цены акций. Этот подход использует классический событийный анализ, который бдоказал свою надежность и валидность в таких работах, Маккинли и Биндер [MacKinlay, 1997; Binder, 1998].

Основная идея подхода: (1) определить, как цена акции должна была изменяться во время релиза Чигры, если бы его (релиза) не было (2) сравнить ожидаемое поведение цены акции с реальным (3) 들ать вывод, является ли эта разница статистически значимой.

\section{Классификаичия релизов игр}

Прежде всего необходимо определить, какие новости для финансового рынка несет в себе тот или иной релиз игры: некоторые из них несут в себе позитивную информацию, другие - негативную, поэтому целесообразно анализировать различные типы событий отдельно. В данной работе тип новости определяется по тому, насколько качество игры соответствует ожидаемому качеству, и рассчитывается по формулам:

Релиз игры считается «хорошим», если

$E M_{i}=\frac{Q_{i}-E\left(Q_{i}\right)}{E\left(Q_{i}\right)}>0.1$

«нормальным», если

$E M_{i}=\frac{Q_{i}-E\left(Q_{i}\right)}{E\left(Q_{i}\right)} \in[-0.1 ; 0.1]$

«плохим», если

$E M_{i}=\frac{Q_{i}-E\left(Q_{i}\right)}{E\left(Q_{i}\right)}<-0.1$

где $F M_{i}-$ процент, на который реальное качество игры і оказалось выше/ниже ожидаемого качества $Q_{i}$ - реальное качество игры і

$E\left(Q_{i}\right)$ - ожидаемое качество игры і

- После использования подобной классификации выясняется, что в выборке присутствует 16 плохих, 30 нормальных и 23 хороших релиза. В дальнейшем эти три группы событий будут анализироваться по отдельности для увеличения точности исследования. 
После дискриминации релизов на три группы появляется необходимость смоделировать, как цена акции должна была себя вести, если бы релиза игры не было. При этом интересно не только поведение в день самого релиза, но и несколько дней до и после него. Группа дней, в которых будет ^рассматриваться поведение цены акции, называется «событийное окно», где 0 - это день релиза. С Событийное окно в данной работе включает в себя 21-й торговый день, то есть рассматриваются 10 亏ддней до релиза игры и 10 дней после.

После определения дней, для которых необходимо смоделировать «нормальное» поведение цены акции, возможен переход непосредственно к самому моделированию. Для этого создается регрессия, которая использует поведение цены акции из «оценочного окна» для прогноза цены в «событийном окне» (см. рис. 1), оценочное окно в данном исследовании равно 50 торговым дням ${ }^{1}$ и зависимой переменной является дневная доходность акции, равная:

$R_{t}=\frac{P_{t}-P_{t-1}}{P_{t-1}}$

×ге $P_{t}$ — цена акции в день $\mathrm{t}$

(9) Регрессия так же включает в себя два типа объясняющих переменных - фактор отраслевого риска - (представлен дневным доходом отраслевого фондового индекса) и фактор риска страны, в которой расположена фирма (представлен дневным доходом фондового индекса страны и дневное процент(не изменение курса домашней валюты к доллару США²). В конечном итоге регрессия имеет следующий вид:

$\sum_{t}^{\text {ol }} R_{t}^{\text {share }}=\alpha+\beta_{1} R_{t}^{\text {industry }}+\beta_{2} R_{t}^{\text {country }}+\left\{\beta_{3} R_{t}^{\text {currency }}\right\}+\varepsilon$,

где $R_{t}^{\text {share }}$ - дневная доходность акции в день $\mathrm{t}$ (один из 50 дней оценочного окна);

$\stackrel{\sim}{\unrhd} R_{t}^{\text {industry }}$ - дневная доходность отраслевого фондового индекса в день t;

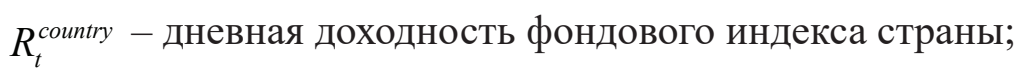

$R_{t}^{\text {currency }}$ - дневное процентное изменение курса домашней валюты к доллару США;

$\varepsilon-$ ошибка.

При подсчете регрессии были предприняты попытки минимизировать количество незначимых параметров и в то же время максимизировать коэффициент детерминации (R-квадрат). Минимальное значение R-квадрата по всем 69 регрессиям - 0,11\%, максимальное - 57,35\%, среднее - 21,16\%. То есть, факторы рисков, описанные выше, объясняют, в среднем, более $20 \%$ дисперсии дневной доходности акции. Однако при интерпретации результатов стоит быть осторожным, так как в некоторых случаях коэффициент детерминации не превышает и $1 \%$, что означает, что для некоторых релизов не удалось достаточно качественно определить механизм изменения цены акции.

Используя коэффициенты, представленные в предыдущем уравнении, рассчитывается ожидаемая дневная доходность в событийном окне для каждого из 21-го дня и для каждого релиза по формуле:

$E\left(R_{t}\right)=\alpha+\beta_{1} R_{t}^{\text {industry }}+\beta_{2} R_{t}^{\text {country }}+\left\{\beta_{3} R_{t}^{\text {currency }}\right\}$.

\section{Тестирование отклонения от ожидаемого поведения ичены акциии}

Теперь необходимо посчитать, как реальное поведение акции отличалось от прогнозируемого (то есть посчитать чрезмерную доходность), и если эта разница будет статистически значима, то будет сделан вывод, что она (разница) была вызвана релизом игры. Чрезмерная дневная доходность рассчитывается по формуле:

$A R_{t}^{n}=R_{t}^{n}-E\left(R_{t}^{n}\right)$

1. Подразумевается, что акция около релиза игры должна себя вести примерно по тем же принципам и законам, что и в предыдущие 50 дней. Иными словами, коэффициенты для каждого из параметров в оценочном окне должны быть равны коэффициентам в событийном окне для каждого релиза.

2. Для всех стран, кроме самих США. 
где $A R_{t}^{n}-$ чрезмерная дневная доходность (Abnormal Return) $i$-й день для игры $n$.

После этого требуется определить, значимо ли отличается средняя дневная чрезмерная доходность от нуля (если релиз игры никак не влияет на поведение акции, то, в среднем чрезмерная дневная ьдоходность должна быть примерно равна нулю). Средняя чрезмерная дневная доходность рассчибывается по формуле для каждого из 21-го дня событийного окна:

$A A R_{t}=\frac{\sum_{n=1}^{69} A R_{t}^{n}}{69}$,

где $A A R_{t}=$ Средняя чрезмерная дневная доходность (Average Abnormal Return)

в $i$-й день.

После того как AAR для каждого дня посчитан, значимость его отличия от нуля тестируется с помощью t-критерия Стьюдента. Выдвигаемые гипотезы таковы:

$H_{0}: A A R_{t}=0$

$H_{1}: A A R_{t} \neq 0$

Результатьл

З3начения AAR и результаты тестов для всех игр и типов событий представлены в таблице 5.

Можно заметить, что единственная значимая AAR была выявлена лишь для 3-го дня до плохого р.леза. Результат неубедителен - из 84 AAR лишь одно значимое, что могло произойти случайно. ऽПоэтому можно сделать вывод, что не были найдены достаточные доказательства, чтобы утверж¿дать, что в какой-либо из дней чрезмерная доходность больше/меньше нуля.

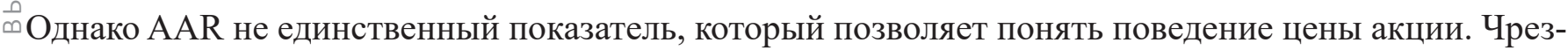
мерные доходности, полученные в течение определенного промежутка времени, можно просуммировать и понять, какую общую чрезмерную доходность получит инвестор, владея активом в заданный временной промежуток. Эта мера называется кумулятивной чрезмерной доходностью (далее - CAR) и рассчитывается по формуле:

$C A R_{t}^{n}=\sum_{i=-t}^{+t} A R_{i}^{n}$

где $C A R_{t}^{n}$ - кумулятивная чрезмерная доходность от владения актива $\mathrm{n}$ в интервале $\mathrm{c}-t$ до $+t^{1}$

$A R_{i}^{n}$ - чрезмерная доходность от владения актива $n$ в $i$-й день до/после релиза игры

Для того чтобы определить, является ли CAR значимо отличимой от нуля, необходимо использовать t-тест для среднего CAR для каждого периода (так же как было сделано для AR).

Было принято решение выбрать три интервала размерами 5-й, 11-й и 21-й торговый день (что примерно равно одной, двум и четырем календарным неделям соответственно). Значения CAAR и их статистическая значимость представлены в таблице 6. Для большей визуализации значения CAAR для «всех», «хороших», «нормальных» и «плохих» релизов представлены на графиках 1, 2, 3 и 4

Из таблицы можно сделать вывод, что, покупая акцию разработчика/издателя за два дня до релиза любой игры (вне зависимости от ее реального и ожидаемого качества) и продавая ее через два дня после релиза, инвестор получит в среднем статистически значимую чрезмерную доходность, равную 1,83\%. Однако, если инвестор будет вкладывать деньги более обдуманно и применять подобную стратегию только при релизе «хороших» игр, его/ее доход увеличится более чем в два раза и станет $3,96 \%$.

Для релиза обычных и «плохих» игр не было выявлено статистически значимой чрезмерной доходности. Также для временного интервала в 21-й день ни один CAAR не оказался значимым, что может указывать на то, что цена акции разработчика/издателя компьютерных игр приходит в равно-

1. Интервалы обычно выбираются симметричными, а день события $\mathrm{t}=0$ находится ровно посередине 
весие быстрее, чем за две недели после релиза.

\section{Рекомендации для инвесторов}

Данная методология кажется более «репрезентативной», чем предыдущая, поэтому инвесторам сто○ит уделить ее результатам особое внимание. Однако стоит понимать, что она также более сложная и не может принять во внимание такие финансовые показатели, как, например, годовое изменение

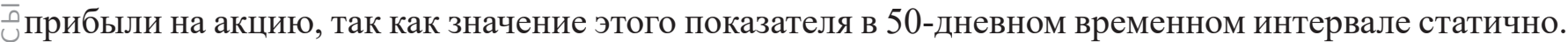

Результаты данной методологии немного похожи на предыдущие. Инвесторам стоит приобретать акции за пару дней до релиза игры, а затем продавать их через два дня после него. Для инвестора штак же будет выгодно заранее определять, будет ли игра «хорошей», и применять вышеописанную

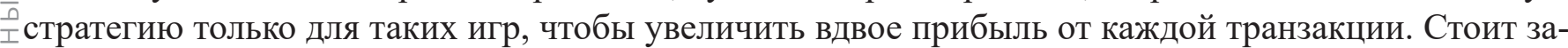
$\leq$ метить, что период, в течение которого можно получить чрезмерную прибыль, весьма ограничен ұля 21-дневного интервала даже средняя кумулятивная чрезмерная доходность для «хороших» игр не оказалась значимой, поэтому инвестору нужно реагировать на события быстро, чтобы получить прибыль.

\section{Недостатки и допущения}

 ладает несколькими недостатками, которые могут быть исправлены в следующих исследованиях. ๓Были выявлены три основных недостатка: проблема выборки, оценка ожидаемого и реального кағчества игры и ошибка спецификации модели.

В изначальную выборку было включено 18 фирм. Однако возможно, что некоторые малые фирмы не были включены в нее из-за того, что они просто не были обнаружены при поиске. Поэтому мож气 но утверждать, что выборка была получена удобным для исследователя образом, а не случайным, что формально запрещает обобщать результаты данной работы. Кроме того, второе ограничение, наложенное на фирмы для включения их в выборку, вероятно, исключит из нее маленькие фирмы с неликвидными акциями, в то время как третье ограничение, наоборот, исключит крупнейшие фирмы. Первое же ограничение для игр исключит из выборки непопулярные игры (без обзоров). Все эти ограничения уменьшают внешнюю валидность исследования.

Оценка ожидаемого и реального качества игр также может быть подвержена критике. Во-первых, нет никаких доказательств, что средняя экспертная оценка показывает истинное качество игры. Сан [Sun, 2012] утверждает, что мнения экспертов обычно сильно поляризованы, что делает вариацию оценок экспертов выше вариации истинного качества игр. Ситуминг и соавторы [Situmeang et al., 2014] подмечают, что эксперты достаточно консервативны и не привыкли ставить слишком разные оценки для игр из одной серии - только очень большое отклонение от обычного качества может заставить их изменить свое мнение, что, наоборот, уменьшает вариацию оценок. Во-вторых, нет никаких доказательств, что ожидания о качестве игры формируются именно по модели адаптивных ожиданий с коэффициентом дисконтирования, равным 0,5. Имеющаяся модель ожиданий также не включает множество параметров, которые потенциально могут влиять на ожидания игроков. Поэтому данный подход к оценке ожидаемого и реального качества неточен, он уменьшает внутреннюю валидность и сокращает мощность тестов.

Последний важный недостаток заключается в выборе контрольных переменных. Вполне возможно, что в модель не были включены некоторые важные параметры. Например, Френч [French, 1980] и Лим с соавторами [Lim et al., 2010] доказали наличие эффекта сезонности в цене акции, а именно доходность акции в понедельник значительно ниже, чем в другие дни, поэтому было бы разумно добавить в основную регрессию фиктивную переменную «Понедельник» ${ }^{1}$. Поэтому, возможно, модель в нынешнем виде некорректно специфицирована, что уменьшает точность полученных результатов.

Существует несколько второстепенных недостатков. Во-первых, была проигнорирована разница 1. Это не было сделано из-за трудоемкости процесса. К тому же мнения о наличии так называемого «Эффекта понедельника» весьма разнятся в академической среде. 
между разработчиками и издателями компьютерных игр (уменьшает точность исследования). Вовторых, нельзя быть уверенным, что результаты, полученные в данной работе, будут применимы в будущем, учитывая инновационный характер индустрии видеоигр (уменьшает внешнюю валидность). В-третьих, в исследовании предполагается, что информация о качестве игры становится пдоступной только после релиза игры, что не является правдой, так как для многих игр выпускаются бета-версии, с помощью которых достаточно точно можно оценить качество конечного продукта -(уменьшает репрезентативность теста).

\section{Заключение}

В данной работе были найдены доказательства, что выпуск игры (особенно той, качество которой превысило ожидания) в среднем положительно влияет на цену акции. Данный эффект длится в отечение нескольких дней после релиза. Однако, принимая во внимание недостатки исследования, будет разумным перепроверить данный результат перед применением или, по крайней мере, использовать его для прогнозирования цены акции только известного разработчика/издателя, получающего доходы в основном от продаж видеоигр, после релиза только популярных игр.

\section{Список используемой литературы}

1. Collier, P. M., 2012. Accounting for managers: interpreting accounting information for decisionmaking. 4th edition, John Wiley \&Sons Ltd

2. Oliver, R. L., 2009. Satisfaction: A behavioral perspective on the consumer. New York: McGrawHill.

3. Anderson, R. E., 1973. Consumer dissatisfaction: The effect of disconfirmed expectancy on perceived product performance. Journal of Marketing Research, 10(1), 38-44.

4. Ball, R., Brown, P., 1968. An Empirical Evaluation of Accounting Income Numbers. J. Acc. Res., Autumn 1968, 6(2), pp. 159-78.

5. Binder, J. J., 1998. The Event Study Methodology Since 1969. Review of Quantitative Finance and Accounting, 11, pp. 111-137

6. Erdem, T., 1998. An empirical analysis of umbrella branding. Journal of Marketing Research, 35(August), 339-351.

7. Fama, E. F., French, K.R., 1992. The Cross-section of Expected Stock Returns. Journal of Finance, 47, pp. 427-465

8. Fama, E. F., French, K.R., 1993. Common risk factors the returns on stock and bonds. Journal of Financial Economics, 33, pp. 3-56.

9. French, K.R., 1980. Stock returns and the weekend effect. Journal of Financial Economics vol. 8, pp. 55-69

10. Kim, J. H., Shamsuddin, A., 2008. Are Asian stock markets efficient? Evidence from new multiple variance ratio tests. Journal of Empirical Finance, 15 (2008), pp. 518-532

11. King, B. F., 1966. Market and Industry Factors in Stock Price Behavior. Journal of Business, 39 (supplement), pp. 139-190.

12. Lakonishok, J., Shleifer, A., Vishny, R. W., 1994. Contrarian Investment, Extrapolation, and Risk. The Journal of Finance, vol. XLIX, (5), pp. 1541-1578

13. Lim, S.Y., Mun Ho, C., Dollery, B., 2010. An empirical analysis of calendar anomalies in the Malaysian stock market. Applied Financial Economics, 20, pp. 255-264

14. Lo, A. W., MacKinlay, A. C., 1988. Stock Market Prices Do Not Follow Random Walks: Evidence from a Simple Specification Test. The Review of Financial Studies, vol. 1, (1), pp. 41-66

15. Lo, A. W., MacKinlay, A. C., 1997. Maximizing predictability in the stock and bond markets. Macroeconomic Dynamics, 1, pp. 102-134.

16. MacKinlay, A. C., 1997. Event studies in economics and finance. Journal of Economic Literature 
Vol. XXXV (March 1997), pp. 13-39

17. Plucker, J. A., Kaufman, J. C., Temple, J. S., Qian, M., 2009. Do experts and novices evaluate movies the same way? Psychology and Marketing, 26(5), 470-478.

18. Poterba, J. M., Summers, L. H., 1988. Mean Reversion in Stock Prices. Journal of Financial Economics, vol. 22, pp. 27-59

19. Salehi, M., Khodadadi, V., Abdolkhani, H., 2011. Forecasting stock price using artificial neural networks: A multi-layer perception model - Iranian evidence. Scientific Research and Essays Vol. 6(19), pp. 4029-4038

20. Scholes, M. S., 1972. The Market for Securities: Substitution Versus Price Pressure and the Effects of Information Share Prices. Joumal of Business 45, pp. 179-211.

21. Scholtens, B., Wang, L., 2008. Oil Risk in Oil Stocks. The Energy Journal; 2008; 29.

22. Situmeang, F. B. I., Leenders, M. A. A. M., Wijnberg, N. M., 2013. The good, the bad and the variable: How evaluations of past editions influence the success of sequels. European Journal of Marketing, 33(3)

23. Situmeang, F. B. I., Leenders, M. A. A.M, Wijnberg, N. M., 2014. History matters: The impact of reviews and sales of earlier versions of a product on consumer and expert reviews of new editions. European Management Journal 32 (2014), pp.73-83

24. Sun, M., 2012. How does variance of product ratings matter? Management Science, 58(4), pp. 696-707.

25. Activision Blizzard, Inc., официальный веб-сайт 2014. Доступен по ссылке: www. activisionblizzard.com

26. Baker, L. B., 2011. Shares of video game companies swing on reviews. Доступен по ссылке: http://www.reuters.com/article/2011/09/16/us-videogame-reviews idUSTRE78F52320110916

27. Bloomberg, 2014. Доступен в Bloomberg terminal, Cass Business School

28. Сарсоm Co., Ltd., официальный веб-сайт, 2014. Доступен по ссылке: www.capcom.com

29. CD Projekt S.A. официальный веб-сайт, 2014. Доступен по ссылке: www.cdprojekt.com

30. Deloitte, 2007. Most consumers read and rely on online reviews; companies must adjust. Доступен по ссылке: www.marketingcharts.com/interactive/most-consumersread-and-rely-on-onlinereviews -companies-must-adjust-2234/.

31. Funcom Productions A/S, официальный веб-сайт, 2014. Доступен по ссылке: www.funcom. $\mathrm{com} /$

32. Hearn, B., Piesse, J., Strange, R., 2010. Market Liquidity and Stock Size Premia in Emerging Financial Markets: The Implications for Foreign Investment. Доступен по ссылке: http://ssrn. com/abstract $=1163777$

33. Majesco Entertainment, официальный веб-сайт, 2014. Доступен по ссылке: www.majescoent. $\mathrm{com} /$

34. Metacritic, 2014, официальный веб-сайт, 2014. Доступен по ссылке: www.metacritic.com

35. NCSOFT, 2014, официальный веб-сайт, 2014. Доступен по ссылке: www.ncsoft.com

36. Square Enix Holdings Co., Ltd., официальный веб-сайт, 2014. Доступен по ссылке: www. hd.square-enix.com

37. Take-Two Interactive Software, Inc, официальный веб-сайт, 2014. Доступен по ссылке: www. take2games.com/

38. THQ Inc., официальный веб-сайт, 2014. Доступен по ссылке: www.thq.com 


\begin{tabular}{|c|c|c|}
\hline b & Номер & Название фирмы \\
\hline & 1 & Activision Blizzard, Inc. \\
\hline I & 2 & Capcom Co., Ltd. \\
\hline$\underline{1}$ & 3 & CD Projekt S.A. \\
\hline$\theta$ & 4 & Funcom Productions A/S \\
\hline 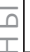 & 5 & Majesco Entertainment \\
\hline$\stackrel{\oplus}{\ulcorner}$ & 6 & NCSOFT \\
\hline 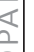 & 7 & Square Enix Holdings Co., Ltd. \\
\hline ᄃ & 8 & Take-Two Interactive Software, Inc. \\
\hline & 9 & THQ Inc. \\
\hline
\end{tabular}

Таблица 2

Рассмотренные компьютерные игры

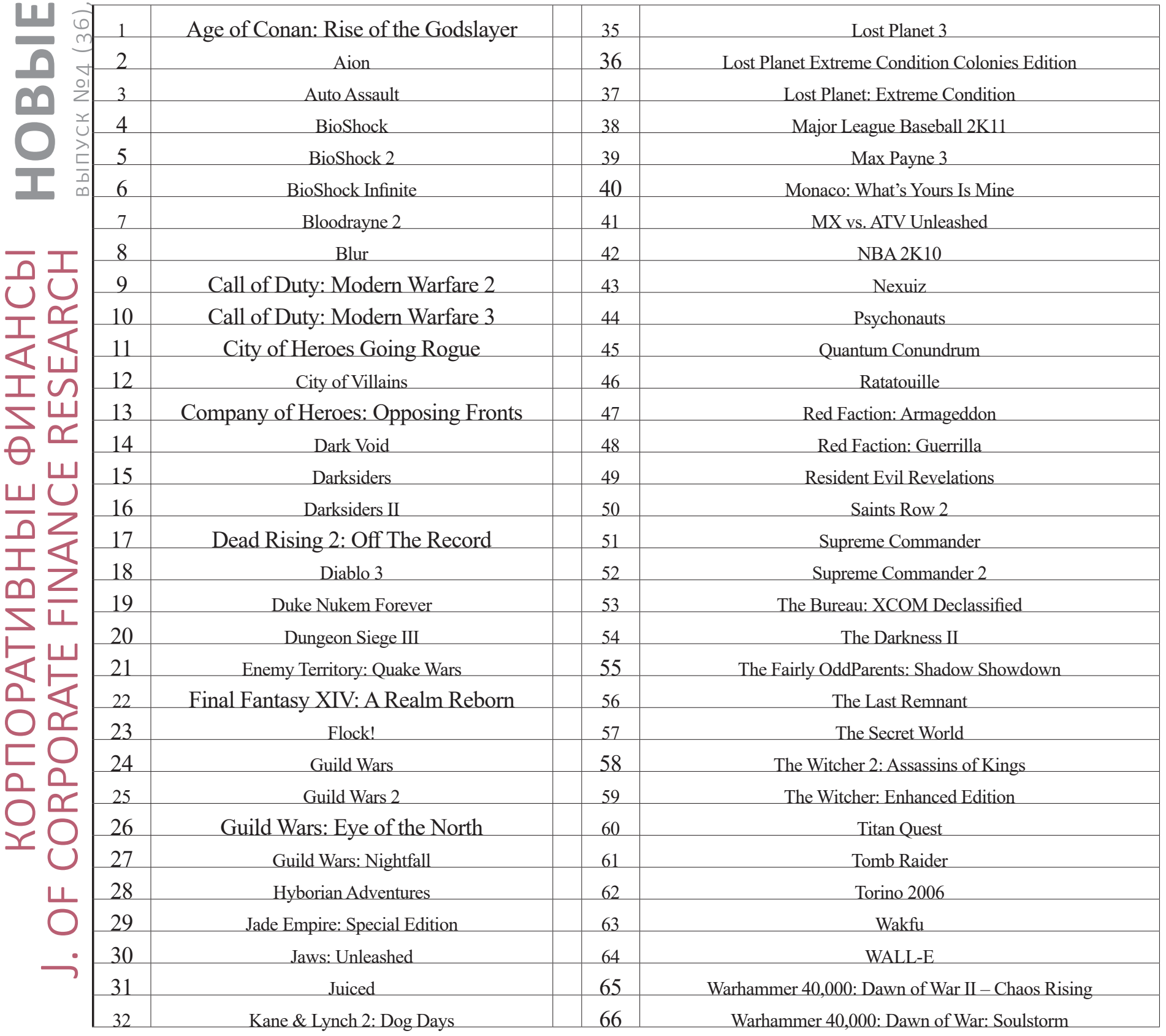




\begin{tabular}{|c|c|c|c|c|}
\hline 33 & Kung Fu Panda & 67 & Warhammer 40,000: Dawn of War: Winter Assault \\
\hline 34 & Lost Planet 2 & 68 & Warhammer 40,000: Space Marine \\
\hline & & 69 & X-Men Origins: Wolverine \\
\hline
\end{tabular}

Таблица 3

Полный список рассмотренных параметров с ссылками на литературу

\begin{tabular}{|c|c|c|}
\hline $\begin{array}{l}\text { Сокращение для на- } \\
\text { звания параметра }\end{array}$ & Название параметра & $\begin{array}{c}\text { Примеры работ, где рассматривался данный } \\
\text { параметр }\end{array}$ \\
\hline ret_cum & Кумулятивный дневной доход акции & (Зависимая переменная) \\
\hline indret & Дневной доход отраслевого фондового индекса ${ }^{I}$ & King $(1966)^{2}$ \\
\hline Cntret & Дневной доход фондового индекса страны ${ }^{3}$ & $\begin{array}{l}\text { Fama, Fisher at.al. (1969), Scholes (1972), } \\
\text { Scholtens \& Wang(2008) }\end{array}$ \\
\hline prev ret & $\begin{array}{c}\text { Кумулятивный доход акции в предыдущем календарном } \\
\text { месяце }\end{array}$ & $\begin{array}{c}\text { Lo \& McKinlay (1988, 1997), Poterba \& Sum- } \\
\text { mers (1988) }\end{array}$ \\
\hline PEchange & $\begin{array}{c}\text { Ежегодное изменение коэффициента “цена/прибыль на } \\
\text { акцию” }\end{array}$ & Salehi et. al (2011) \\
\hline Pbkchange & Ежегодное изменение Р/В коэффициента фирмы & $\begin{array}{c}\text { Fama \& French }(1992,1993), \text { Scholtens \& } \\
\text { Wang (2008) }\end{array}$ \\
\hline Capchange & Ежегодное изменение капитализации фирмы & $\begin{array}{c}\text { Fama \& French }(1992,1993), \text { Hearn, Piesse, } \\
\text { Strange (2010) }\end{array}$ \\
\hline Equitychange & Ежегодное изменение собственного капитала фирмы & Salehi et. al (2011) \\
\hline EPSchange & Ежегодное изменение коэффициента "прибыль на акцию" & Salehi et. al (2011) \\
\hline Quality & Реальное качество игры & - \\
\hline Expect & Ожидаемое качество игры & - \\
\hline Exp_met & Процент оправданности ожиданий (Quality/ Expect - 1) & - \\
\hline Expindex & $\begin{array}{c}\text { Индекс оправданности ожиданий (Quality-Expect)/(Qual- } \\
\text { ity+ Expect) }\end{array}$ & - \\
\hline Good & Фиктивная переменная. =1 если Exp_met $>0.1$ & - \\
\hline $\mathrm{Bad}$ & Фиктивная переменная. $=1$ если Ехр met $<-0.1$ & - \\
\hline
\end{tabular}

1. В качестве показателя ситуации в индустрии был использован индекс S\&P Global 1200 Information Technologies Index.

2. Автор не изучал важность биржевого индекса, но доказал, что остатки, которые дает рыночная модель, коррелированы для фирм из одной индустрии. Это позволяет предполагать, что включение данного параметра может быть полезным.

3. Для оценки ситуации на финансовом рынке страны происхождения рассматриваемой фирмы были использованы следующие биржевые индексы: CША - S\&P 500, Япония - Nikkei-225, Польша - WIG20, Норвегия - OSEBX, Южная Корея - KOSPI.. 
Результаты регрессии

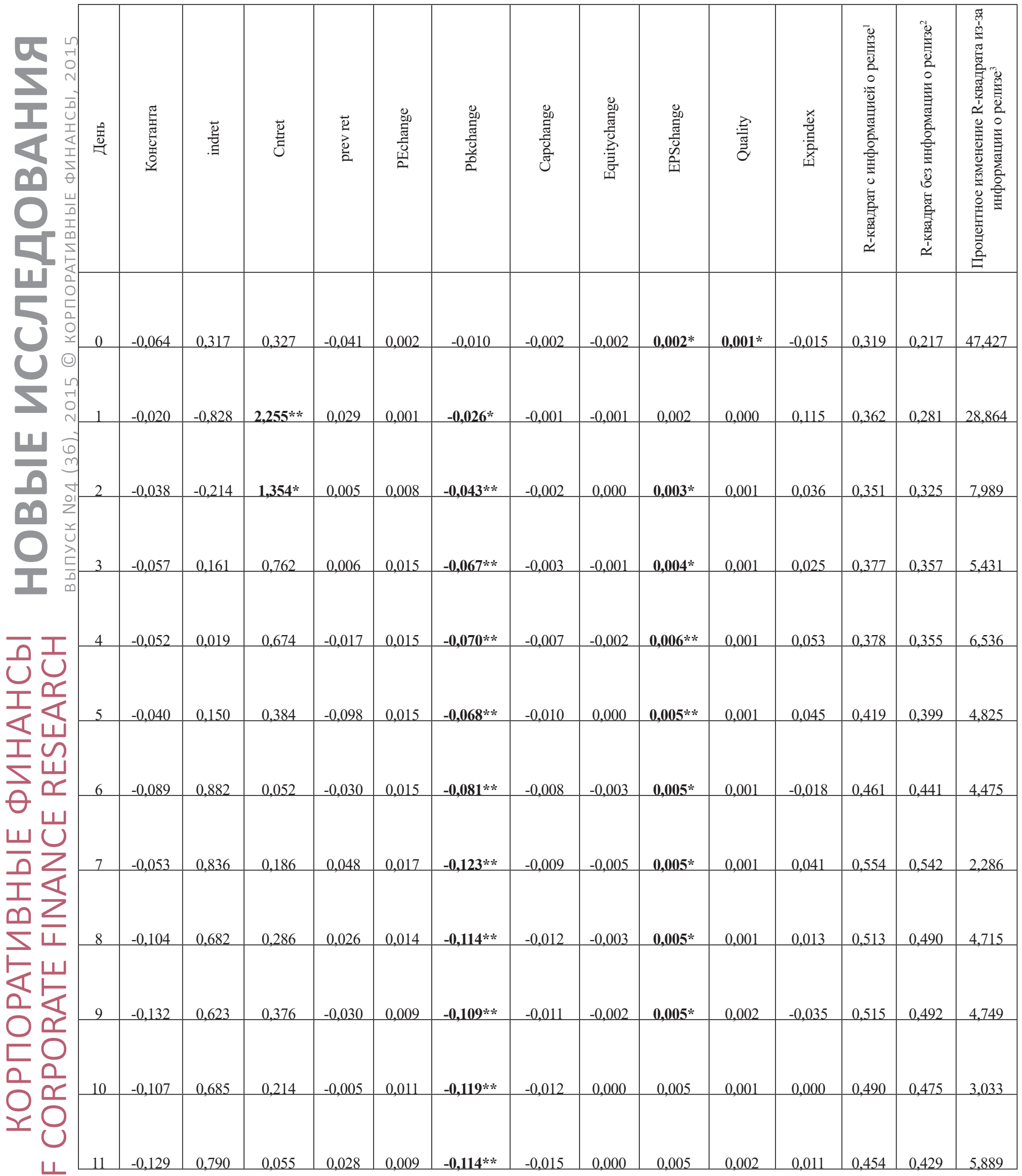

1. Коэффициент детерминации (R-квадрат), когда в модель включены параметры ожидаемого и реального качества игры.

2. Коэффициент детерминации (R-квадрат), когда в модель не включены параметры ожидаемого и реального качества игры.

3. Насколько первый коэффициент детерминации больше второго (в \%). 


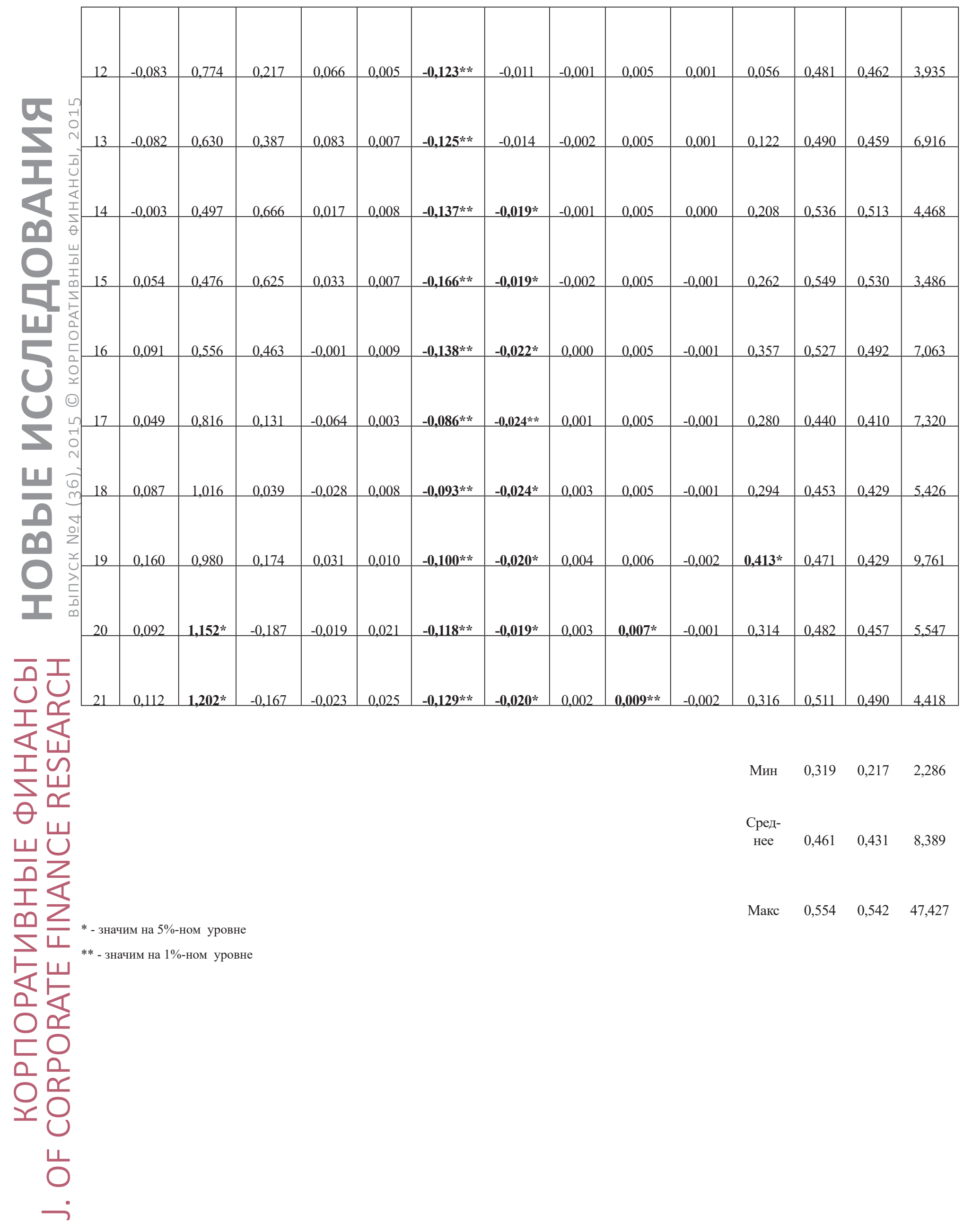




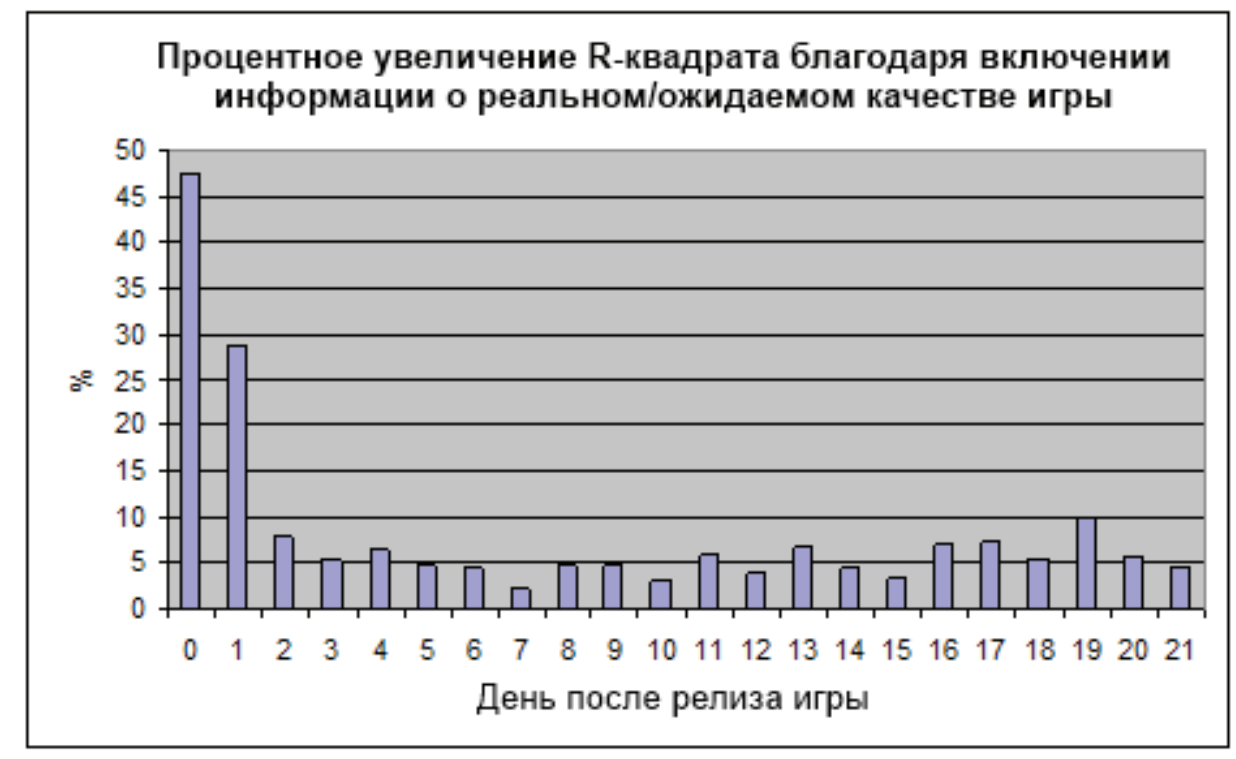

Гистограмма 1

Таблииа 5

Значения средних чрезмерных дневных доходностей и их статистическая значимость

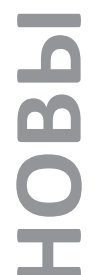

\begin{tabular}{|c|c|c|c|c|}
\hline \multicolumn{5}{|c|}{ Средняя чрезмерная доходность (АAR) } \\
\hline
\end{tabular}

* - значим на 5\%-ном уровне,

** - значим на $1 \%$-ном уровне

Средняя кумулятивная чрезмерная доходность и ее статистическая значимость

\begin{tabular}{|c|c|c|c|c|}
\hline \multicolumn{5}{|c|}{ Средняя кумудятивная чрезмерная доходность (САAR) } \\
\hline Размер интервала & Обшее & Хороший & Нормальный & Плохой \\
\hline 21 & $1,59 \%$ & $5,52 \%$ & $-1,29 \%$ & $1,37 \%$ \\
\hline 11 & $2,35 \%$ & $6,90 \% * *$ & $0,22 \%$ & $-0,19 \%$ \\
\hline
\end{tabular}


* с значим на 5\%-ном уровне,

** - значим на 1\%-ном уровне

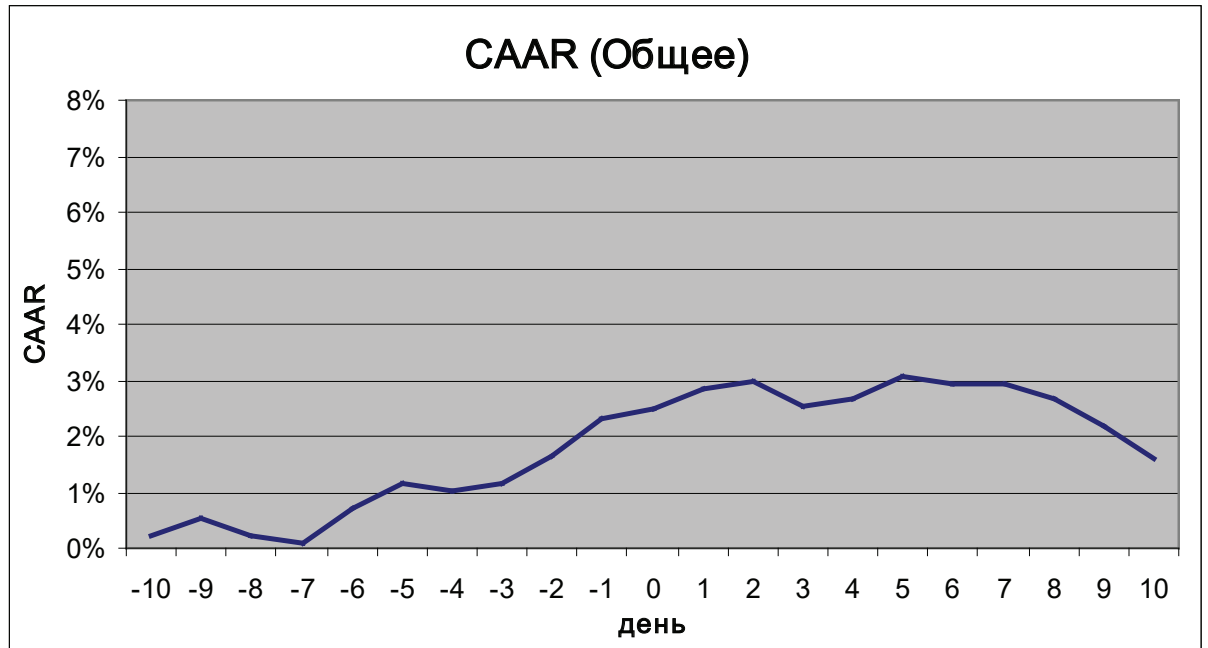

График 1. Средняя кумулятивная чрезмерная доходность для всех релизов

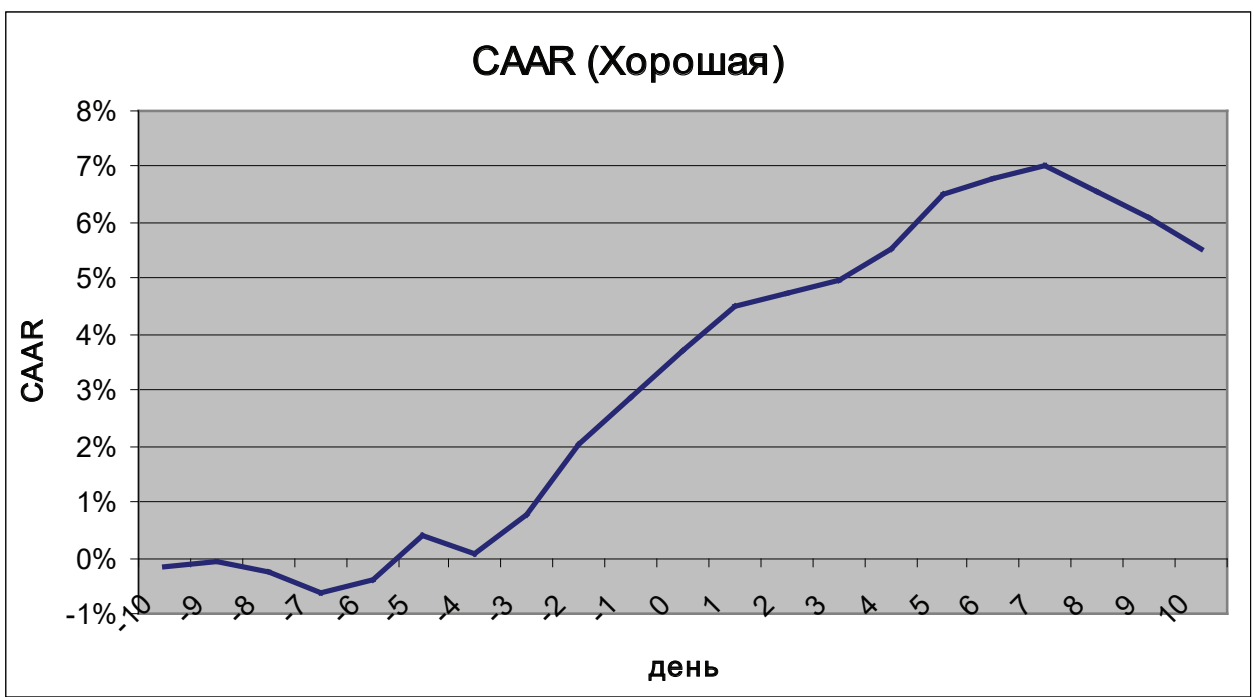

График 2. Средняя кумулятивная чрезмерная доходность для хороших релизов

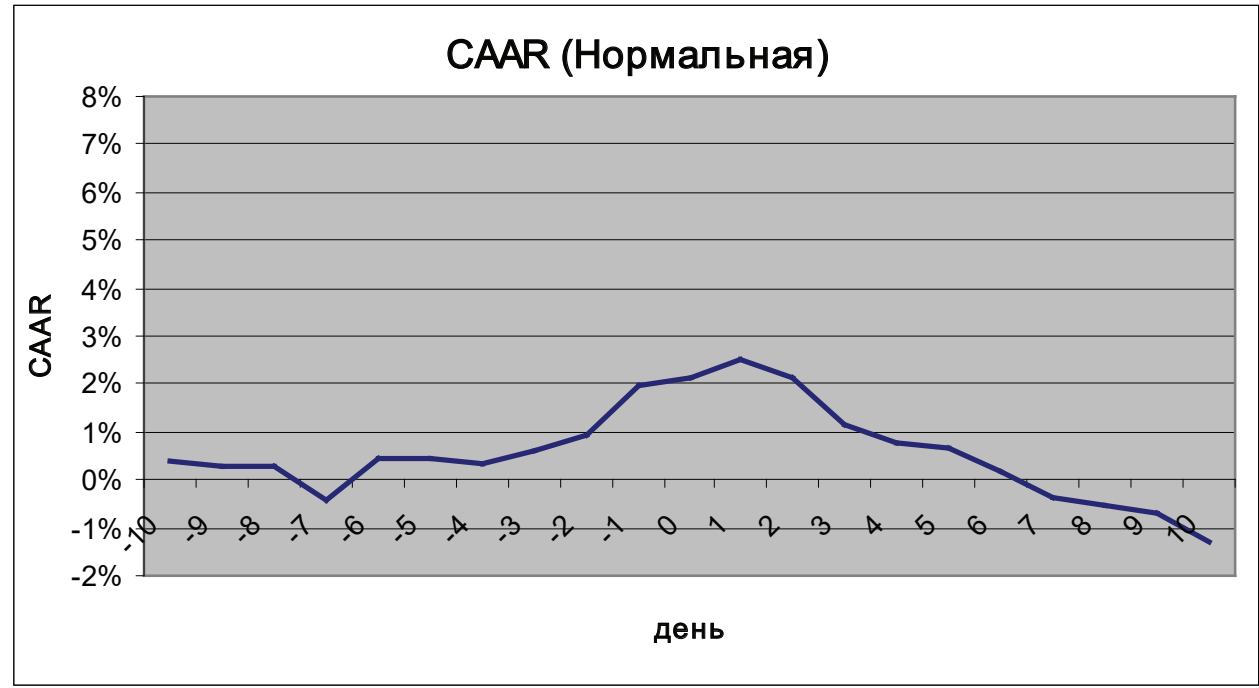

График 3. Средняя кумулятивная чрезмерная доходность для нормальных релизов 


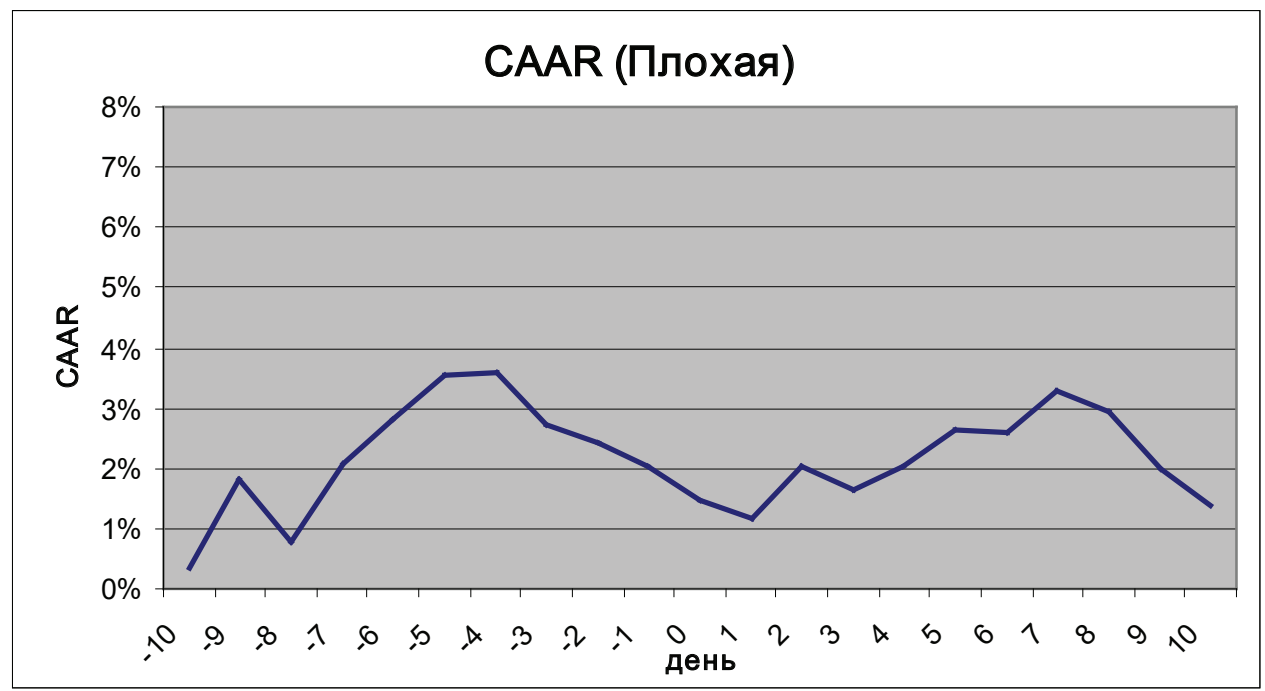

График 4. Средняя кумулятивная чрезмерная доходность для плохих релизов

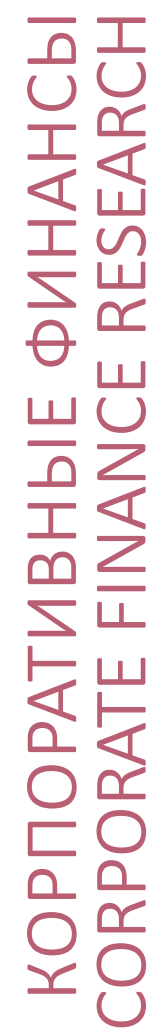

늠 


\title{
THE IMPACT OF THE FINAL PRODUCT TO THE SHARE
}

PRICE OF THE MANUFACTURER

\author{
Alexander Solomatin, \\ Non-state educational institution «Moscow international higher business school \\ «IRBIS» (Institute), Pro-rector on academic research
}

Jaroslav Solomatin, Closed joint stock company Commercial Bank «Citibank», DCC Analyst

\section{Abstract}

In this work, we study how the release of the product to the market (release of computer game in this case) may affect the share price of its developer/publisher.

Financial data were obtained for 9 public companies - computer games' developers/publishers, which satisfied three predetermined criteria. Then all games developed/published by the firms were examined and 69 games published during period from 2004 to 2013 were selected in accordance with three other criteria. (-) After that the data were processed by two methodologies. First methodology is just a set of ordinary linear ${ }_{\rightarrow}$ regressions, in which cumulative daily return of developers'/publishers' shares is a dependent variable and financial performance of the corresponding firm, industry and country; as well as real and expected quality iof published game; are independent variables. Second methodology is one of the types of event study mmethods, in which abnormal return and cumulative abnormal return of the developers'/publishers' shares $\checkmark$ are dependent variables. In both cases for estimation of real quality of the game average expert score from ${ }_{2}$ Metacritic website was used, whereas expected quality was modeled using adaptive expectations method, which took into account real quality of previous games of corresponding developer/publisher.

Results of the first method claim, that information about game release and its quality improves quality of the share price's forecast on more than $25-45 \%$, but only for two days after the release. Results of the second method state, that release of any game causes a short-term abnormal increase in share price on $1,83 \%$, and release of a "good game" causes 3,96\% increase. The results can be used by investors to receive additional return and by producers' management bodies to understand better the factors, which affect the value of their companies. They also allow to conclude that release of the product (computer game) has a positive short-term impact on share price of the producer, especially if the quality of the product turned out to be higher than expected.

Keywords: Price, stock, profitability, securities market, product, capitalization

JEL: G11

\section{References}

1. Collier, P. M., 2012. Accounting for managers: interpreting accounting information for decisionmaking. 4th edition, John Wiley \&Sons Ltd

2. Oliver, R. L., 2009. Satisfaction: A behavioral perspective on the consumer. New York: McGrawHill.

3. Anderson, R. E., 1973. Consumer dissatisfaction: The effect of disconfirmed expectancy on perceived product performance. Journal of Marketing Research, 10(1), 38-44.

4. Ball, R., Brown, P., 1968. An Empirical Evaluation of Accounting Income Numbers. J. Acc. Res., Autumn 1968, 6(2), pp. 159-78.

5. Binder, J. J., 1998. The Event Study Methodology Since 1969. Review of Quantitative Finance and Accounting, 11, pp. 111-137

6. Erdem, T., 1998. An empirical analysis of umbrella branding. Journal of Marketing Research, 35(August), 339-351.

7. Fama, E. F., French, K.R., 1992. The Cross-section of Expected Stock Returns. Journal of Finance, 


\section{7, pp. 427-465}

8. Fama, E. F., French, K.R., 1993. Common risk factors the returns on stock and bonds. Journal of Financial Economics, 33, pp. 3-56.

9. French, K.R., 1980. Stock returns and the weekend effect. Journal of Financial Economics vol. 8, pp. 55-69

10. Kim, J. H., Shamsuddin, A., 2008. Are Asian stock markets efficient? Evidence from new multiple variance ratio tests. Journal of Empirical Finance, 15 (2008), pp. 518-532

11. King, B. F., 1966. Market and Industry Factors in Stock Price Behavior. Journal of Business, 39 (supplement), pp. 139-190.

12. Lakonishok, J., Shleifer, A., Vishny, R. W., 1994. Contrarian Investment, Extrapolation, and Risk. The Journal of Finance, vol. XLIX, (5), pp. 1541-1578

13. Lim, S.Y., Mun Ho, C., Dollery, B., 2010. An empirical analysis of calendar anomalies in the Malaysian stock market. Applied Financial Economics, 20, pp. 255-264

14. Lo, A. W., MacKinlay, A. C., 1988. Stock Market Prices Do Not Follow Random Walks: Evidence from a Simple Specification Test. The Review of Financial Studies, vol. 1, (1), pp. 41-66

15. Lo, A. W., MacKinlay, A. C., 1997. Maximizing predictability in the stock and bond markets. Macroeconomic Dynamics, 1, pp. 102-134.

16. MacKinlay, A. C., 1997. Event studies in economics and finance. Journal of Economic Literature Vol. XXXV (March 1997), pp. 13-39

17. Plucker, J. A., Kaufman, J. C., Temple, J. S., Qian, M., 2009. Do experts and novices evaluate movies the same way? Psychology and Marketing, 26(5), 470-478.

18. Poterba, J. M., Summers, L. H., 1988. Mean Reversion in Stock Prices. Journal of Financial Economics, vol. 22, pp. 27-59

19. Salehi, M., Khodadadi, V., Abdolkhani, H., 2011. Forecasting stock price using artificial neural networks: A multi-layer perception model - Iranian evidence. Scientific Research and Essays Vol. 6(19), pp. 4029-4038

20. Scholes, M. S., 1972. The Market for Securities: Substitution Versus Price Pressure and the Effects of Information Share Prices. Joumal of Business 45, pp. 179-211.

21. Scholtens, B., Wang, L., 2008. Oil Risk in Oil Stocks. The Energy Journal; 2008; 29.

22. Situmeang, F. B. I., Leenders, M. A. A. M., Wijnberg, N. M., 2013. The good, the bad and the variable: How evaluations of past editions influence the success of sequels. European Journal of Marketing, 33(3)

23. Situmeang, F. B. I., Leenders, M. A. A.M, Wijnberg, N. M., 2014. History matters: The impact of reviews and sales of earlier versions of a product on consumer and expert reviews of new editions. European Management Journal 32 (2014), pp.73-83

24. Sun, M., 2012. How does variance of product ratings matter? Management Science, 58(4), pp. 696-707.

25. Activision Blizzard, Inc., официальный веб-сайт 2014. Доступен по ссылке: www. activisionblizzard.com

26. Baker, L. B., 2011. Shares of video game companies swing on reviews. Доступен по ссылке: http://www.reuters.com/article/2011/09/16/us-videogame-reviews idUSTRE78F52320110916

27. Bloomberg, 2014. Доступен в Bloomberg terminal, Cass Business School

28. Сapcom Co., Ltd., официальный веб-сайт, 2014. Доступен по ссылке: www.capcom.com

29. CD Projekt S.A. официальный веб-сайт, 2014. Доступен по ссылке: www.cdprojekt.com

30. Deloitte, 2007. Most consumers read and rely on online reviews; companies must adjust. Доступен по ссылке: www.marketingcharts.com/interactive/most-consumersread-and-rely-on-online- 
reviews -companies-must-adjust-2234/.

31. Funcom Productions A/S, официальный веб-сайт, 2014. Доступен по ссылке: www.funcom. com/

32. Hearn, B., Piesse, J., Strange, R., 2010. Market Liquidity and Stock Size Premia in Emerging Financial Markets: The Implications for Foreign Investment. Доступен по ссылке: http://ssrn. com/abstract $=1163777$

33. Majesco Entertainment, официальный веб-сайт, 2014. Доступен по ссылке: www.majescoent. com/

34. Metacritic, 2014, официальный веб-сайт, 2014. Доступен по ссылке: www.metacritic.com

35. NCSOFT, 2014, официальный веб-сайт, 2014. Доступен по ссылке: www.ncsoft.com

36. Square Enix Holdings Co., Ltd., официальный веб-сайт, 2014. Доступен по ссылке: www. hd.square-enix.com

37. Take-Two Interactive Software, Inc, официальный веб-сайт, 2014. Доступен по ссылке: www. take2games.com/

38. THQ Inc., официальный веб-сайт, 2014. Доступен по ссылке: www.thq.com 